\title{
Evaluation of the best single-energy scanning in energy spectrum CT in lower extremity arteriography
}

\author{
GUOKUN WANG ${ }^{*}$, DELI ZHAO ${ }^{*}$, ZAISHENG LING, HAIBO WANG, SHANSHAN YU and JINLING ZHANG \\ Department of Computed Tomography, The Second Affiliated Hospital of Harbin Medical University, Harbin, \\ Heilongjiang 150086, P.R. China
}

Received October 30, 2018; Accepted April 26, 2019

DOI: $10.3892 /$ etm.2019.7666

\begin{abstract}
The present study aimed to apply the best single-energy (SE) scanning in energy spectrum computed tomography (CT) to evaluate the usefulness of lower extremity arterial angiography imaging in patients with lower extremity arterial occlusive disease. A total of 64 patients diagnosed with lower extremity arterial occlusive disease were randomly selected and divided into either the experimental group $(n=32)$ or the control group $(n=32)$. The two treatment groups were scanned for lower extremity arteriography using the best SE scanning mode of energy spectrum CT Gemstone imaging (GSI) and mixed energy scanning mode of multi-slice helical CT (MSCT). The CT images, image noise, contrast-to-noise ratio (CNR) and quality scores of the images of lower extremity arteries between the two groups were compared. Image quality of the two experimental groups were independently evaluated by two imaging diagnostic physicians. The CT scores and CNR of the lower extremity arteries were significantly higher in the experimental group compared with the control group $(\mathrm{P}<0.01)$. No statistically significant differences in the background noise between the two groups were observed $(\mathrm{P}<0.05)$. The image quality scores of two groups, with the differences between the two diagnosticians, were found to be statistically significant $(\mathrm{P}<0.01)$. In the lower extremity arterial angiography, the image quality of the best SE in the CT GSI scanning mode was observed to be superior to that taken using MSCT mixed energy scanning mode.
\end{abstract}

Correspondence to: Dr Jinling Zhang, Department of Computed Tomography, The Second Affiliated Hospital of Harbin Medical University, 246 Xuefu Road, Nangang, Harbin, Heilongjiang 150086, P.R. China

E-mail: zjinlzjl@126.com

${ }^{*}$ Contributed equally

Key words: lower extremity arterial occlusive disease, computed tomography, Gemstone spectral imaging

\section{Introduction}

Changes in dietary habits and an aging population have led to the gradual increase in the incidence of peripheral vascular diseases, with a noteworthy rise in lower extremity arterial occlusive disease (1). The lower limb blood vessels of patients often exhibit severe calcification and stenosis, a condition that hampers the visualization of vascular structures or changes in the vessel branching (1). Accurate and early detection of lower extremity arterial stenosis and collateral circulation can improve disease management by providing patients with timely and appropriate treatment (2). Currently, clinical diagnosis of lower extremity arterial disease is reliant on imaging (2). The most commonly used imaging techniques include lower extremity vascular ultrasound, computed tomography angiography (CTA), magnetic resonance angiography and digital subtraction angiography (DSA) (2). The application of DSA is adjudged to be the 'gold standard' for the assessment of lower extremity arterial pathologies (1). However, DSA-based analyses are invasive, and it is highly likely that computerized tomography (CT) lower extremity arterial angiography will become the procedure of choice. In comparison with conventional CT lower extremity arterial angiography, CT Gemstone Spectral Imaging (GSI), which functions by imaging under alternating high and low voltage conditions, provides higher image quality (3). Therefore, in the present study, the best single-energy (SE) scanning mode, which was achieved by optimising the contrast-to-noise ratio (CNR), and multi-slice spiral CT (MSCT) combined energy-scanning mode were employed to perform lower extremity arterial angiography. The imaging quality of the two modes, along with their relative precision in lower extremity arteriography imaging, were compared and evaluated.

\section{Materials and methods}

Patient information. A total of 64 individuals (39 males; 25 females) were clinically diagnosed with or suspected of lower extremity arterial occlusive disease based on energy spectrum CT lower extremity arterial imaging between December 2015 and December 2016 at The Second Affiliated Hospital of Harbin Medical University (Harbin, China). They were between 41 and 78 years of age with height ranging between 155 and $182 \mathrm{~cm}$, and the body mass index (BMI) ranging between 21.8 and 23.6. Patients were randomly assigned 
to the experimental group (32 patients) or the control group (32 patients). Some of the clinical manifestations included lower limb swelling, low skin temperature, limping, chronic infections, ulcers, gangrene and trauma. Within 1 week all patients were assessed using lower extremity arterial digital subtraction angiography or Doppler ultrasound. This study was designed to be a randomized, controlled study, with all patients undergoing CTA lower extremity arterial imaging. The inclusion criteria were: i) $\geq 18$ years of age, clinically diagnosed with or suspected of peripheral arterial occlusive disease; ii) liver and kidney function adjudged to be within the normal range; and iii) no history of allergy to iodinated contrast agents. The exclusion criteria were: i) $\mathrm{BMI} \geq 30 \mathrm{~kg} / \mathrm{m}^{2}$; and ii) inability to undergo CT examination. All patients were required to sign informed consent forms. This study was approved by the Ethics Committee of The Second Affiliated Hospital of Harbin Medical University (Nangang, China).

Examination method. Patients were examined using the Discovery CT-750HD model CT GSI machine (GE Healthcare). Scanning parameters and tube voltage in the experimental group were set to automatic mode. Images were reconstructed using the best electronic voltage measured with an optimised CNR. The control group was treated with mixed energy scanning mode of the MSCT. The scanning tube voltage was $120 \mathrm{kVp}$ and the tube current was $200 \mathrm{~mA}$. All other parameters were maintained constant between the two groups, with only slight variations between individuals: Pitch, 1.375:1; tube rotation speed, $0.8 \mathrm{sec} /$ turn; scanning layer thickness, $5 \mathrm{~mm}$; the abdominal aortic level was set as the monitoring point; and 150 HU was set as the monitoring threshold for the commencement of a trace trigger scan. Delay was set to $6 \mathrm{sec}$. Scanning direction was from the head to the foot. The layer thickness during image reconstruction was $1.25 \mathrm{~mm}$. The contrasting agent $(80-100 \mathrm{ml}$ of $350 \mathrm{mg} / \mathrm{ml}$ iodohydrin) was injected at a rate of $4.0 \mathrm{ml} / \mathrm{sec}$, followed by the delivery of 20-40 $\mathrm{ml}$ physiological saline.

Image processing. Original data obtained from the $\mathrm{CT}$ machine were first transmitted to the image post-processing workstation. The GSI image of the experimental group was calculated using a post-processing software (GSI-view software, volume share 5; GE Healthcare) to process the SE image of the best electron perturbation based on the maximum CNR of the blood vessel/muscle. The best electronic voltage was determined to be in the range of $55-60 \mathrm{keV}$. The three-dimensional images of the lower extremity arteries from both experimental and control groups were reconstructed using Multi-Planner Reformation, Volume Rendering, Maximum Intensity Projection and Curve Reformation. Two senior physicians independently examined the images using a double-blinded method for all directions and angles. In incidences where their diagnoses were not in agreement, DSA and Doppler ultrasound results were compared, and further consultations took place until mutual agreements were reached.

Image quality evaluation. The following parameters of the lower extremity arteries were included: Lower extremity arterial course, distribution, shape, width and length of the lesion, with or without stenosis, occlusion and deformities, the presence of collateral circulation, vascular wall changes and
Table I. Comparison of participants from two groups.

\begin{tabular}{lccc}
\hline Parameters & Experimental & Control & P-value \\
\hline Age & $54 \pm 5$ & $58 \pm 4$ & $>0.05$ \\
Sex (male/female) & $18 / 14$ & $21 / 11$ & $>0.05$ \\
Height & $164 \pm 6.3$ & $166 \pm 5.5$ & $>0.05$ \\
BMI & $22.5 \pm 1.27$ & $21.3 \pm 2.16$ & $>0.05$
\end{tabular}

BMI, body/mass index.

vascular artifacts. The iliac arteries, the superficial femoral artery, the popliteal artery and the anterior tibial artery were evaluated. Two imaging diagnostic doctors, using a double-blinded method, were recruited to perform a subjective evaluation of the images. Evaluation of image quality was performed using a five-point system (4), with all images fixed in the same window width and position, applying the following specific scoring criteria: 5 points, excellent image; 4 points, good image; 3 points, intermediate image; 2 points, poor image and 1 point, very poor.

Image analysis. In this study, 22 patients in the experimental group were subjected to CTA and DSA. As DSA may present risks and is expensive, only the patients with severe clinical symptoms and those that needed interventional treatment underwent the procedures; 22 of the 32 patients required this intervention. The lower extremity arteries were divided into eight anatomical segments: Common iliac artery, internal iliac artery, external iliac artery, femoral artery, popliteal artery, anterior tibial artery, posterior tibial artery and fibular (peroneal) artery. The degree of blood stenosis in coronary artery stenosis was assessed using a four-tier assessment system (5): Mild stenosis, $<50 \%$; moderate stenosis and luminal stenosis, $50 \%-75 \%$; severe stenosis and tube cavity stenosis, $>75 \%$; and complete occlusion, $100 \%$. Stenosis $=[$ (stenosis near the proximal end of the normal diameter of the vessel-stenosis of the diameter of the vessel)/stenosis near the proximal end of the normal vascular diameter] $x 100$. The diameter of the vessel was measured was to assess stenosis. In cases of multiple stenoses observed in the same vascular segment, the highest level of stenosis was used for comparison. A total of 28 lower extremity arterial CTA and DSA from similar vascular segments of 22 patients were compared.

Statistical analysis. All data were analyzed using the SPSS20.0 software package (IBM Corp.). Count index was presented as percentage, and measurement index was presented as mean \pm standard deviation. $\chi^{2}$ test was used for comparisons of sex, whereas independent samples t-test was used for comparisons of age, height, weight and objective image quality. Mann-Whitney U-test was applied for rating subjective image quality. The sensitivity and specificity of vascular stenosis were calculated using the $95 \%$ confidence interval. Consistency between the two groups was analyzed using the $\kappa$-test, and $\mathrm{P}<0.05$ was considered to indicate a statistically significant difference. 
Table II. Comparison of image quality (objective) from two participant groups.

\begin{tabular}{lccr}
\hline Parameters & Experimental & Control & P-value \\
\hline Iliac Arteries CT Value (HU) & $525.4 \pm 54.1$ & $386.2 \pm 61.3$ & $<0.05$ \\
Superficial Femoral arteries CT Value (HU) & $488.6 \pm 53.2$ & $352.1 \pm 41.4$ & $<0.05$ \\
Popliteal Arteries CT Value (HU) & $410.6 \pm 63.7$ & $320.4 \pm 58.1$ & $<0.05$ \\
Anterior Tibial arteries CT Value (HU) & $352.2 \pm 75.4$ & $230.5 \pm 68.2$ & $<0.05$ \\
Background Noise & $9.15 \pm 1.12$ & $10.27 \pm 1.24$ & $>0.05$ \\
Contrast Noise Ratio & $53.8 \pm 9.5$ & $34.7 \pm 8.6$ & $<0.05$ \\
\hline
\end{tabular}

HU, Hounsfield unit.

\section{Results}

General comparison. No significant differences were identified in relation to sex, age, height and weight between patients in the experimental and control groups (Table I).

Image quality evaluation (Objective). The best electron emission images reconstructed using GSI were more uniform compared with the conventional MSCT model. The arterial image was uniform, the edge of the vessel was smooth, the small branch was well defined, the artifact was minimal, the background noise $(\mathrm{BN})$ was low and the CNR was greatly improved. The CT values of the contrast agent in the iliac, superficial femoral, popliteal and anterior tibial arteries were better compared with those derived from the MSCT mixed energy model. The CT value and CNR of the contrast agent were observed to be significantly higher in the iliac, femoral, popliteal and anterior tibial arteries of the experimental group compared with the control group $(\mathrm{P}<0.05)$. The CNR of the MSCT hybrid energy scanning mode was improved, and the image quality was better than that of the MSCT hybrid energy-scanning model. No significant difference was identified for $\mathrm{BN}$ between the two groups ( $\mathrm{P}>0.05$; Table II).

Image quality evaluation (Subjective). Evaluation of the quality of the scanned images was independently performed by two imaging diagnostic physicians. The total image quality score from physician 1 was 155 for the experimental and 133 for the control groups, whereas physician 2 scored 154 for the experimental and 134 for the control groups (Table III). There was also a high degree of consistency between the physicians' evaluations of the image quality. The evaluation demonstrated that the image quality in the experimental group was better compared with that of the control group. The arterial image was uniform, the edge of the vessel was smooth, the small branch was well defined, the artifact was minimal, the BN was low and the CNR was greatly improved. Experimental images showed the vascular wall changes more clearly and the vascular artifacts were markedly increased (Figs. 1 and 2).

Comparative analyses of CTA and DSA in diagnosing lower limb arterial stenosis. CTA and DSA were applied to the lower extremity arteries of 22 patients in the experimental group. A total of 352 blood vessels were applied in the comparative analyses of CTA and DSA described in the present study.
A summary of the specific distribution of vascular stenosis levels was presented in Table IV. Of the 352 blood vessels analyzed, 311 scored consistently for the degree of stenosis in blood vessels using the two diagnostic techniques applied; with the diagnostic accuracy rate of $94.03 \%$ (331/352). In incidences of misdiagnosis, three moderate stenoses of the blood vessels were underestimated by the CTA as mild stenosis, two segments of severe stenosis of the CTA were underestimated as moderate stenosis, four mild stenoses of the vascular CTA were overestimated as moderate stenosis, seven segments of moderate stenosis of the vascular CTA were overestimated as severe stenosis and five segments of severe stenosis of the vascular CTA were overestimated as occlusion. The sensitivity of CTA in the diagnosis of lower extremity arterial stenosis $(\geq 50 \%)$ was $93.18 \%(205 / 220)$ with the specificity at $95.45 \%(126 / 132)$ compared with the diagnostic 'gold standard' DSA. The application of CTA and DSA for the diagnosis of lower extremity arterial stenosis was determined to be reliable $(\kappa=0.927)$, with a high degree of consistency. Therefore, the analyses performed in the present study suggest that both CTA imaging and DSA can be used as a reliable means of diagnosing lower extremity arterial stenosis.

\section{Discussion}

Atherosclerosis is a systemic vascular disease. Lower extremity arterial occlusive disease is caused by a number of conditions, including distal limb ischemia, nutritional disorders, limb ulceration and necrosis (6). Arterial CTA has long been applied as the most direct and effective method for the diagnosis of arteriosclerosis of the lower extremity; as it can visualize the lower extremity arteries to accurately display the intraluminal contrast agent through the vessel wall calcification (6). These features enable the accurate and reliable diagnosis of lower extremity arterial disease and the development of treatment strategies. The ability of CTA to determine the formation of distal collateral circulation in the lower extremity arterial wall is superior to that of conventional DSA angiography and ultrasonography $(7,8)$.

In the MSCT coupled with mixed energy imaging system currently in clinical use, the tube produced by the X-ray system has a certain spectral width of a multi-chromatogram (9). It generates photons at a spectrum of energy levels that can penetrate the human body. Low-energy rays can be easily 
absorbed whereas high-energy rays readily pass through the body (9). Therefore, the average energy of the rays tends to increase during the propagation, causing the rays to become harder (9). This beam-hardening effect can result in severe beam hardening artifacts (BHA) that may compromise image quality and diagnostic assessment. By contrast, the SECT GSI image utilizes an SE level of X-ray through the object after image acquisition. This effectively removes the beam hardening effects, leading to more accurate CT values, improved image quality and better detection of small lesions $(10,11)$. Conventional CT equipment cannot project an SE X-ray. The energy spectrum CT with an X-ray tube that instantaneously switches between high- and low-energy imaging modes can generate mixed energy images. The SE image of 40-140 keV can be reconstructed using energy spectrum analysis (12). At an SE level, the difference between the set point of interest and the physical organ can be maximized, whilst the noise value can be minimized. The clinical application of energy spectrum $\mathrm{CT}$ is based primarily on the following four technical characteristics: Elimination of hardening artifacts, optimization of image quality and CNR, quantitative analysis of the matter and comprehensive analysis of energy spectrum $(13,14)$. In the present study, the optimized image quality and CNR was first determined to be used in the energy spectrum synthesis analysis to reconstruct a single contrast image with the best CNR. Compared with the images produced using mixed energy $\mathrm{CT}$, those produced using the best electron micrographs were uniform. The vascular and branch anatomical structures were imaged with high precision, and the lesions were revealed to be much more evident.

The aim of the present study was to investigate whether the GSI is superior to the MSCT in the visualization of the anatomical details of the lower extremity arteries, the contrast between blood vessels and surrounding tissues and the optical amenability of the vascular lesions for diagnosis. The best SE energy map in the experimental group was in the $55-60 \mathrm{keV}$ range, in which blood vessels and muscle exhibited the largest CNR. Outside of this range, the CNR was reduced. The lower the X-ray energy level, the higher the contrast $\mathrm{CT}$ value and hence greater the difference between blood vessels and the surrounding tissue CT. By contrast, CNR was lower and BN was higher in the control group. Higher X-ray energy levels resulted in smaller BN, in turn causing a sharp drop in the CT value of the contrast agent and a decrease in CNR. Therefore, the function of the best SE graph was to find a balance between the CT difference and BN, optimizing the CNR leading to the best $\mathrm{keV}$ (15). Results from the present study demonstrated that the best electron emission images reconstructed using GSI were more uniform compared with the conventional MSCT model. The arterial image was uniform, the edge of the vessel was smooth, the small branch was well defined, the artifact was minimal, the BN was low and the CNR was greatly improved. The CT values of the contrast agent in the iliac, superficial femoral, popliteal and anterior tibial arteries were better compared with those derived from the MSCT mixed energy model. The results demonstrated that the CNR of the MSCT hybrid energy scanning mode was improved, and the image quality was better than that of the MSCT hybrid energy-scanning model. This was consistent with the subjective evaluation results. 
Table IV. Assessment of lower extremity arterial stenosis from 44 segments using CTA and DSA.

DSA (segment)

\begin{tabular}{lcccccc}
\cline { 2 - 6 } CTA (segment) & Healthy & Mild stenosis & Moderate stenosis & Severe stenosis & Complete occlusion & Total \\
\hline Healthy & 73 & 0 & 0 & 0 & 0 & 73 \\
Mild stenosis & 0 & 57 & 3 & 0 & 0 & 60 \\
Moderate stenosis & 0 & 4 & 79 & 2 & 0 & 85 \\
Severe stenosis & 0 & 0 & 7 & 83 & 0 & 90 \\
Complete occlusion & 0 & 0 & 0 & 5 & 39 & 44 \\
Total & 73 & 61 & 89 & 90 & 39 & 352 \\
\hline
\end{tabular}

CTA, computed tomography angiography; DSA, digital subtraction angiography.

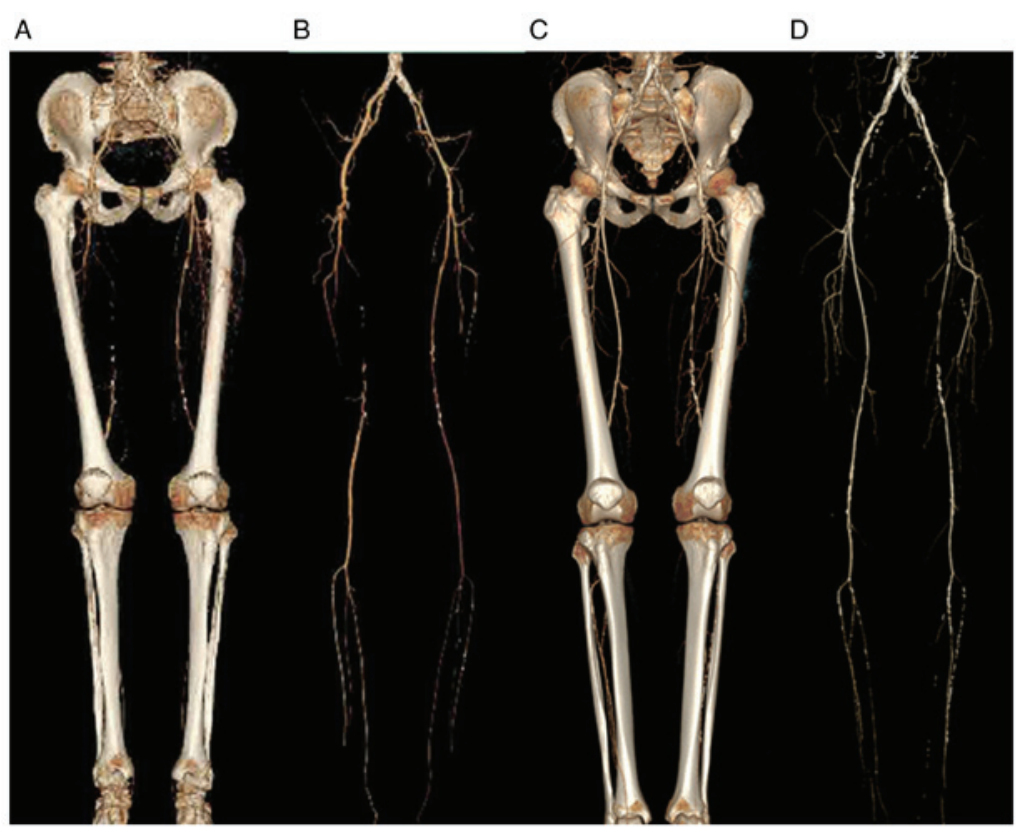

Figure 1. Virtual reconstruction of images of the lower extremity arteries from one patient from each group with and without the bones. The courses of lower extremity arteries were all normal, parts of the tube wall not smooth, the lumen filling was mostly good, part of the lumen was narrow and a calcified plaque has formed. (A and B) Image constructed using energy hybrid power of MSCT (A) with bone and (B) without bone in a patient in the control group. (C and D) Image constructed using SECT GSI (C) with bone and (D) without bone in a patient in the experimental group. The coccyx and sacrum were removed in order to show the vessels more clearly. The image quality of that taken using SECT GST is superior to that taken using the energy hybrid power of MSCT. CT, computerized tomography; GSI, Gemstone imaging; SECT, single energy CT; MSCT, multi-slice helical CT.

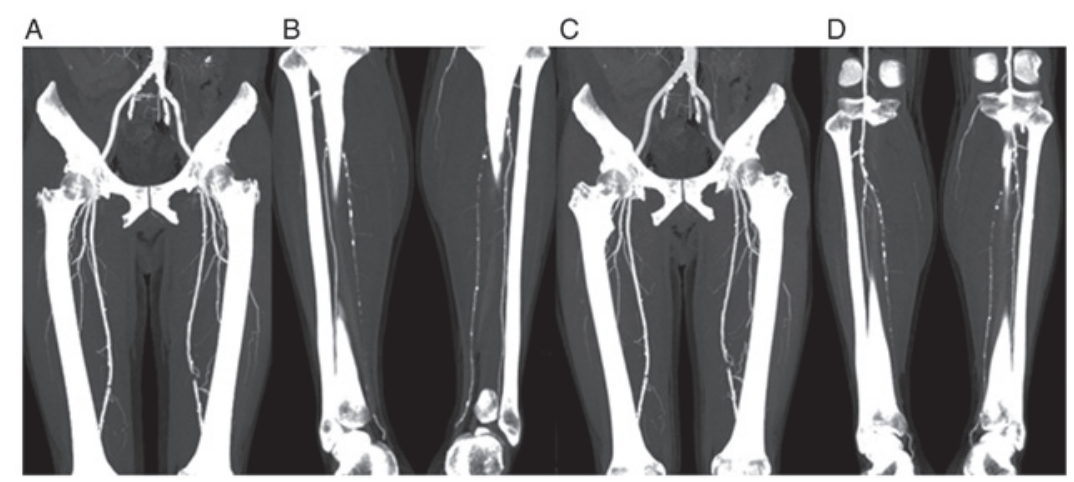

Figure 2. Maximum intensity projection images of the same patients illustrated in Fig. 1. The lower extremity arteries exhibited multiple calcified plaque and stenosis, with images taken using SECT revealed the arterial branches and details superior to that taken using the energy hybrid power of GSI MSCT. (A) Energy hybrid power of MSCT images of the upper part of the lower extremity arteries and (B) the lower part of the lower extremity arteries in a patient. (C) SECT GSI images of the upper part of lower extremity arteries and (D) of the lower part of the lower extremity arteries in another patient. CT, computerized tomography; MSCT, multi-slice helical CT; SE, single energy CT; GSI, Gemstone imaging. 
As patients with lower extremity arterial occlusive disease often exhibit additional underlying pathological conditions, including hypertension and diabetes, the need to strictly control for the amount of contrast agent is crucial (16). SECT GSI imaging can alter the contrast agent $\mathrm{CT}$ value and CNR, and can be performed in the absence of a contrast agent to offer a certain degree of remediation. Therefore, SECT GSI imaging, without affecting the diagnostic criteria, uses a lower dosage of contrast agent by adjusting the energy value of the SE energy map. This has important clinical consequences, as it reduces the side effects associated with contrast agents. In addition, SECT GSI imaging has the potential to reduce metallic artifacts and BHA (17), which is helpful for the clearer display of stenting in patients undergoing lower extremity arterial stent implantation and to provide a theoretical basis for assessing restenosis after stenting.

DSA has long been considered as the 'gold standard' for the diagnosis of vascular lesions; it can readily detect the dynamic vascular changes in addition to the underlying pathological alterations, all of which are crucial in the treatment of vascular lesions (1). Although DSA is invasive and often results in trauma, it is still considered as an indispensable tool for the diagnosis and treatment of lower limb vascular disease (7). Patients with lower extremity arteriosclerosis obliterans often manifest varying degrees of cardiovascular and cerebrovascular disease in other peripheral parts of the vascular stenosis, thus increasing the risk of DSA-based examination and surgery (7). By contrast, CTA is a safe and effective imaging technique for imaging lower limb vascular lesions (18). It entails a high temporal and spatial resolution, features that are important for identifying the location, extent, degree of stenosis and collateral circulation, in addition to occlusion of the distal arterial trunk (1). For patients with DSA results as part of the diagnostic criteria, the two methods provided consistent conclusions on the blood vessels in a total of 311 segments, the diagnostic accuracy of $94.03 \%$ (331/352), CTA diagnosis of lower extremity arterial stenosis $(\geq 50 \%)$ has a sensitivity $93.18 \%(205 / 220)$ and a specificity of $95.45 \%$ (126/132), indicating that CTA diagnosis of lower extremity arterial stenosis parallels that of DSA. These results are consistent with the literature $(3,18)$. In the present study analysis, there were 14 incidences of CTA overestimation and 5 incidences of underestimation of stenosis. Further examination of these vascular segments revealed that the extent of stenosis severity was either too high or too low for a number of reasons. Examples include overestimated vascular segments with more severe wall calcification, post-treatment methods improperly applied in some cases, severe vascular stenosis or completed occlusion, weak vascular enhancement and the eccentric stenosis of the blood vessel wall caused by some errors. Therefore, CTA image post-processing must incorporate three-dimensional images with the axial image from the original combination of observation techniques. In addition, appropriate adjustments to the window width and window position are imperative for reducing errors in determining vascular stenosis or occlusion lesions.

There are a number of limitations associated with this study. The sample size was relatively small, so that the results could have been biased, which can affect the accuracy of the statistical results to a certain extent. In addition, the contrast agent dosage and injection rate were not adjusted to body weight indexes of participants, and this might have an impact on the degree of vascular enhancement. A subjective selection of vascular region of interest position, vascular CT value, BN and CNR might have been chosen in a biased fashion. Lastly, radiation dose measurements were not performed between the two groups of participants.

In conclusion, this study demonstrated that CT GSI lower extremity arteriography is a clinically feasible approach and that the best SE scanning mode image quality is superior to the image quality of MSCT mixed energy scanning mode. CTA imaging is of clinical significance in the screening of patients with clinically suspected lower extremity arteriosclerosis obliterans, as it facilitates correct diagnosis and may lead to more effective therapeutic interventions.

\section{Acknowledgements}

Not applicable.

\section{Funding}

The present study was supported by the National Science Foundation of China (grant no. 81701772).

\section{Availability of data and materials}

All data generated or analyzed during the present study are included in this published article.

\section{Authors' contributions}

GKW, DLZ and JLZ conceived of and designed the experiments, and wrote and revised the manuscript. GKW, DLZ, ZSL, SSY, and JLZ performed the experiments and analysed the data. HBW also performed the experiments.

\section{Ethics approval and consent to participate}

The present study was approved by the Ethics Committee of The Second Affiliated Hospital of Harbin Medical University (Nangang, China).

\section{Patient consent for publication}

All patients were required to sign informed consent forms.

\section{Competing interests}

The authors declare that they have no competing interests.

\section{References}

1. Heijenbrok-Kal MH, Kock MC and Hunink MG: Lower extremity arterial disease: Multidetector CT angiography meta-analysis. Radiology 245: 433-439, 2007.

2. Lin XZ, Miao F, Li JY, Dong HP, Shen Y and Chen KM: High-definition CT Gemstone spectral imaging of the brain: Initial results of selecting optimal monochromatic image for beam-hardening artifacts and image noise reduction. J Comput Assist Tomogr 35: 294-297, 2011.

3. Kang MJ, Park CM, Lee CH, Goo JM and Lee HJ: Focal iodine defects on color-coded iodine perfusion maps of dual-energy pulmonary CT angiography images: A potential diagnostic pitfall. AJR Am J Roentgenol 195: W325-W330, 2010. 
4. Pache G, Krauss B, Strohm P, Saueressig U, Blanke P, Bulla S, Schäfer O, Helwig P, Kotter E, Langer M and Baumann T: Dual-energy CT virtual noncalcium technique: Detecting posttraumatic bone marrow lesions-feasibility study. Radiology 256 : 617-624, 2010.

5. Lim S, Shin H, Lee Y, Yoon JW, Kang SM, Choi SH, Park KS, Jang HC, Choi SI and Chun EJ: Effect of metabolic syndrome on coronary artery stenosis and plaque characteristics as assessed with 64-detector row cardiac CT. Radiology 261: 437-445, 2011.

6. Mueller T, Hinterreiter F, Poelz W, Haltmayer M and Dieplinger B: Mortality rates at 10 years are higher in diabetic than in non-diabetic patients with chronic lower extremity peripheral arterial disease. Vasc Med 21: 445-452, 2016.

7. Pollak AW, Norton PT and Kramer CM: Multimodality imaging of lower extremity peripheral arterial disease: Current role and future directions. Circ Cardiovasc Imaging 5: 797-807, 2012.

8. Kock MC, Dijkshoorn ML, Pattynama PM and Myriam Hunink MG: Multi-detector row computed tomography angiography of peripheral arterial disease. Eur Radiol 17: 3208-3222, 2007.

9. Bowden RG: Contrast nephropathy: Risk factors and the role of beta blockers. Anatol J Cardiol 15: 241, 2015.

10. Danad I, Fayad ZA, Willemink MJ and Min JK: Recent advances in cardiac computed tomography: Dual energy, spectral and molecular CT imaging. JACC Cardiovasc Imaging 8: 710-723, 2015.

11. Obaid DR, Calvert PA, Gopalan D, Parker RA, West NJE, Goddard M, Rudd JHF and Bennett MR: Dual-energy computed tomography imaging to determine atherosclerotic plaque composition: A prospective study with tissue validation. J Cardiovasc Comput Tomogr 8: 230-237, 2014.
12. Danad I, Hartaigh BÓ and Min JK: Dual-energy computed tomography for detection of coronary artery disease. Expert Rev Cardiovasc Ther 13: 1345-1356, 2015.

13. Arnoldi E, Lee YS, Ruzsics B, Weininger M, Spears JR, Rowley CP, Chiaramida SA, Costello P, Reiser MF and Schoepf UJ: CT detection of myocardial blood volume deficits: Dual-energy CT compared with single-energy CT spectra. J Cardiovasc Comput Tomogr 5: 421-429, 2011.

14. Takahashi N, Vrtiska TJ,Kawashima A,Hartman RP,Primak AN, Fletcher JG and McCollough CH: Detectability of urinary stones on virtual nonenhanced images generated at pyelographic-phase dual-energy CT. Radiology 256: 84-190, 2010.

15. Yao Y, Ng JM, Megibow AJ and Pelc NJ: Image quality comparison between single energy and dual energy CT protocols for hepatic imaging. Med Phys 43: 4877, 2016.

16. He J, Ma X, Wang Q, Fan J and Sun Z: Spectral CT demonstration of the superior mesenteric artery: Comparison of monochromatic and polychromatic imaging. Acad Radiol 21: 364-368, 2014.

17. Lee JS and Chen JC: A single scatter model for X-ray CT energy spectrum estimation and polychromatic reconstruction. IEEE Trans Med Imaging 34: 403-1413, 2015.

18. Li YH, Peng XG, Jin H, Zhao GF, Ding RR, Wei HL, Lu ZX and Deng G: The improvement of imaging quality of lower extremity angiography with optimal single energy image of spectral CT. J Intervent Radiol 25: 997-1001, 2016. 